\title{
Mitogen-Activated Protein Kinase Kinase 3 Is Required for Regulation during Dark-Light Transition
}

\begin{abstract}
Horim Lee*
Plant growth and development are coordinately orchestrated by environmental cues and phytohormones. Light acts as a key environmental factor for fundamental plant growth and physiology through photosensory phytochromes and underlying molecular mechanisms. Although phytochromes are known to possess serine/threonine protein kinase activities, whether they trigger a signal transduction pathway via an intracellular protein kinase network remains unknown. In analyses of mitogen-activated protein kinase kinase (MAPKK, also called MKK) mutants, the mkk3 mutant has shown both a hypersensitive response in plant hormone gibberellin (GA) and a less sensitive response in red light signaling. Surprisingly, light-induced MAPK activation in wild-type (WT) seedlings and constitutive MAPK phosphorylation in dark-grown mkk3 mutant seedlings have also been found, respectively. Therefore, this study suggests that MKK3 acts in negative regulation in darkness and in light-induced MAPK activation during darklight transition.
\end{abstract}

\section{INTRODUCTION}

Light is one of the most important environmental factors affecting the physiological and developmental growth of plants, which is the major source of energy for plant life. Phytochrome (phy) is a photoreceptor that senses light signals and regulates lightmediated plant growth. Once phytochromes sense light signals, they translocate immediately from the cytoplasm to the nucleus and make complexes with certain proteins in subnuclear photobodies (speckles), where light-induced phys regulate positive or negative regulatory transcription factors (TFs) of photomorphogenesis (Chen and Chory, 2011; Leivar and Quail, 2011). Positively acting TFs including ELONGATED HYPOCOTYL 5 (HY5), LONG HYPOCOTYL IN FAR-RED 1 (HFR1), and LONG AFTER FAR-RED LIGHT 1 (LAF1) are stabilized by the inhibition of COP1 E3 ubiquitin ligase-mediated degradation

Department of Pre-PharmMed, College of Natural Sciences, Duksung Women's University, Seoul 132-714, Korea

*Correspondence: hrlee1375@ duksung.ac.kr

Received 3 March, 2015; revised 30 March, 2015; accepted 6 April 2015; published online 17 June, 2015

Keywords: Arabidopsis, gibberellin, light, MAP kinase kinase, MAPK cascade
(Jang et al., 2005; Saijo et al., 2003; Seo et al., 2003; Yang et al., 2005). Negatively acting TFs such as PHYTOCHROMEINTERACTING FACTORs (PIFs), which promote skotomorphogenic growth, are degraded through the direct interaction with light-induced phys (Leivar and Quail, 2010). Notably, PIFs have recently been reported to be regulated by other phytohormones such as gibberellin (GA) and brassinosteroid (BR), because PIFs directly interact with DELLA and BZR1 proteins in each signaling pathway, respectively (de Lucas et al., 2008; Feng et al., 2008; Oh et al., 2012), suggesting that PIFs integrate multiple signaling pathways for the downstream morphogenesis (Leivar and Quail, 2010).

Both positive and negative regulatory TFs involved in light signaling are commonly regulated by ubiquitin-proteasomemediated degradation and phosphorylation is one of the important post-translational modifications in this process. Recently, casein kinase II (CK2), a ubiquitous Ser/Thr protein kinase, has been reported to phosphorylate both PIF1 at multiple sites and also the positively acting regulator HFR1 (Bu et al., 2011; Park et al., 2008). Although phosphorylation is known to be crucial to proteasome-mediated degradation for the promotion of positive regulator's stabilities and the repression of negative regulators, the kinases that phosphorylate the regulatory TFs involved in light signaling have not been completely investigated.

The mitogen-activated protein kinase (MAPK) cascade usually functions as an important signaling network involved in physiological and developmental processes in eukaryotes. This MAPK cascade is usually composed of sequential activations of three classes of Ser/Thr protein kinases: 20 MAPK (MPK), 10 MAPK Kinase (MKK), and 60 MAPK Kinase Kinase (MKKK) (Hamel et al., 2006; Ichimura et al., 2002). Since plants cannot move to avoid environmental damage such as biotic and abiotic stresses, they have evolved with complex signaling networks to deal with various environmental challenges. Indeed, plant genomes encode the largest number of MAPK cascade genes in all sequenced eukaryotes (Hamel et al., 2006; Ichimura et al., 2002), suggesting that plant MAPK cascade genes have functional redundancies.

The MAPK Kinase (MKK) gene family has relatively few members in canonical MAPK cascade gene families, which are classified into four groups (A to $D$ ) based on the sequence similarities. MKK1, MKK2, and MKK6 are classified into group A MKK gene family (Hamel et al., 2006). Previous studies showed that MKK1 and MKK2 are activated by various stresses such as wound, cold, drought, and salt (Matsuoka et al., 2002; Teige et al., 2004). Because the mutant phenotypes of the $m k k 1$ mkk2 double mutant are similar to those of $m p k 4$ and 
mekk1, the MEKK1-MKK1/2-MPK4 cascade module was genetically established and this cascade was revealed to negatively regulate innate immune response and ROS signaling (Gao et al., 2008; Pitzschke et al., 2009). MKK6 was identified to activate MPK13 using the functional complementation of mutant yeasts (Melikant et al., 2004) and NQK1, an MKK6 ortholog in tobacco, was revealed to regulate the formation of the cell plate during cytokinesis (Soyano et al., 2003). MKK4 and $M K K 5$ belonging to group $C$ MKK gene family are known to activate MPK3 and MPK6 in the microbe-associated molecular pattern-triggered immune response through the transient expression system in protoplasts (Asai et al., 2002). Several studies also suggest that MKK4 and MKK5, with YDA as an MAPKKK and MPK3 and MPK6 as MAPKs, control the celltype specification in each developmental stage of the stomatal lineage (Wang et al., 2007). More recently, a different YDA cascade module, including MKK7 and MKK9 of group D MKK gene family, was found to show overlapping function with MKK4 and MKK5 in stomatal development (Lampard et al. 2009; 2014). MKK7 and MKK9 were also reported to be involved in auxin and ethylene signaling pathways, respectively (Dai et al., 2006; Yoo et al., 2008).

$M K K 3$ is the only member classified into group $\mathrm{B} M K K$ gene family, and has an unusual gene structure in its C-terminal extension encoding a nuclear transfer factor (NTF) domain (Hamel et al., 2006). Although the biological function of the NTF domain in the MKK3 is not clear, since the unicellular green algae Chlamydomonas contains a single MKK with the NTF domain in the genome, it seems to be evolutionarily conserved in photosynthetic eukaryotes (Hamel et al., 2006). A recent study showed that the MKK3-MPK6 cascade module is activated by jasmonic acid (JA) signals and that this cascade module negatively regulates AtMYC2 in the JA-dependent gene expression (Takahashi et al., 2007). By promoter analysis, MKK3 expression was observed in vascular tissues, and was also induced by Pseudomonas syringae pv tomato strain DC3000 (Pst DC3000) (Dóczi et al., 2007). In transient expression approaches, the MKK3-MPK6 cascade module was activated by flg22 peptides, whereas $\mathrm{H}_{2} \mathrm{O}_{2}$ signals activated MPK7 in an MKK3-dependent manner (Dóczi et al., 2007). Most recently, the MKK3-MPK6-MYC2 cascade module was also revealed to be involved in blue light-mediated signaling (Sethi et al., 2014). Although the involvement of MKK3 in blue light-mediated seedling development has just been revealed, the role of MAPK cascades in light signaling remains largely unclear. Here I describe the MAPK phosphorylation induced by light signals and the repressed MAPK activation by MKK3 in darkness. Since the $m k k 3$ mutant also showed a GA-hypersensitive phenotype, these results suggest the vigorous involvement of MAPK cascades in light and GA signaling pathways.

\section{MATRIALS AND METHODS}

Plant materials and growth conditions Columbia-0 (Col-0) was used as the wild-type (WT) Arabidopsis plant. The mkk3-1 (Salk_051970) mutant was in Col-0 background and was obtained from the Arabidopsis Biological Resource Center (ABRC). Sequence information of genotyping primers of Salk_051970 was obtained from the T-DNA primer design program of SIGnAL (LP: 5'-GAA CAA ACG TTT TCT CAT GTG TG-3'; RP: 5'-AGA AGG ATC CAG ATG CTC GAC$\left.3^{\prime}\right)$. WT and mutant plants were grown in soil at $22^{\circ} \mathrm{C}, 65 \%$ humidity, and $80 \mu \mathrm{mol} \mathrm{m} \mathrm{m}^{-2} \mathrm{~s}^{-1}$ light intensity under intermediate light conditions (12 $\mathrm{h}$ light/12 $\mathrm{h}$ dark photoperiod) in a growth room. For seedling analysis, Arabidopsis seeds were germinated and grown in 6-well plates containing $1 \mathrm{ml}$ of liquid medium [0.5 X Murashige and Skoog (MS) and $0.5 \%$ sucrose, pH 5.8 adjusted with $\mathrm{KOH}$ ] or MS plates containing $0.5 \times \mathrm{MS}, 1 \%$ sucrose, and $0.7 \%$ phytoagar, $\mathrm{pH} 5.8$ adjusted with $\mathrm{KOH}$. To measure hypocotyl length, seedlings were vertically grown in MS plates with/without sucrose for 4 days at red light $(7 \mu \mathrm{mol}$ $\left.\mathrm{m}^{-2} \mathrm{~s}^{-1}\right)$ or dark conditions. For the MAPK analysis during darklight transition, seedlings were grown in liquid medium for 7 days in darkness and then exposed to white light $\left(80 \mu \mathrm{mol} \mathrm{m} \mathrm{m}^{-2} \mathrm{~s}^{-1}\right)$ for 10,30 and $60 \mathrm{~min}$.

\section{RT-PCR analysis}

To harvest tissues, seedlings were instantaneously frozen by liquid nitrogen in $1.5 \mathrm{ml}$ tubes. Frozen tissues were ground using a blue pestle and a motor-driven grinder. Total RNA was isolated from ground powder of tissues with TRIzol reagent (Life Technologies, USA). First strand cDNA was synthesized from $1 \mu \mathrm{g}$ of total RNA with M-MLV reverse transcriptase (Promega, USA). For analyzing the knock-out state in the mkk3-1 mutant, semi-quantitative RT-PCR was performed with WT and mkk3-1 plants using gene-specific primers for the $3^{\prime}$ partial fragment of $M K K 3$ (forward: $5^{\prime}-\mathrm{CAG}$ TTA TTC TTA TCC AGC TG-3' reverse: 5'-AAG CCA AGA TCT TTG AAA CTC GG-3') (Takahashi et al., 2007) and the $5^{\prime}$ partial fragment of MKK3 (forward: 5'-TCA TCT TCC ACA TCT CAA GGA-3'; reverse: 5'-TCT ATG ATT AGG GAT ATG AAT AG-3'). TUB4 (TUBULIN BETA CHAIN 4, At5g44340) was used as a control gene (forward: 5'-ATG AGA GAG ATC CTT CAT ATC C-3'; reverse: 5'-AAT GAA TGA CAC ACT TGG AAA C-3'). All quantitative RT-PCR analyses were performed by StepOne Real-Time PCR System with the Power SYBR Green PCR Master Mix (Applied Biosystems) and specific primers for PIF3 (forward: 5'-GAC GGC GTG ATA GGA TCA ACG A-3'; reverse: 5'-CTG CAC TTG AAG TTG GAG TGA-3'), PIF4 (forward: 5'-GCC AAA ACC CGG TAC AAA ACC A-3'; reverse: 5'CGC CGG TGA ACT AAA TCT CAA CAT C-3'), HY5 (forward: 5'-AAG AGA ACA AGC GGC TGA AG-3'; reverse: 5'-TCT TGC TTG CTG AGC TGA AA-3'), and ELF4 (forward: $5^{\prime}-T T G$ ACC GGA ATT TCA GAC AAG TGC-3; reverse: 5'-TTC GAC ATG TTA TCA GCC ATT CTC G-3'). ACT2 (ACTIN2, At3g18780) was used as a control gene (forward: 5'-TCC CTC AGC ACA TTC CAG CAG AT-3'; reverse: 5'-AAC GAT TCC TGG ACC TGC CTC ATC-3')

In vivo protein kinase assay

For an in-gel kinase assay, seedling extracts containing $300 \mu \mathrm{g}$ of proteins were fractionated in $10 \%$ SDS-PAGE gel containing $0.25 \mathrm{mg} \mathrm{ml}^{-1}$ of myelin basic protein (MBP), a general MAPK substrate. The protein denaturing, renaturing, and kinase activity assay in the gel were performed as previously described (Zhang and Klessing, 1997). For an immunoblot assay, proteins were fractionated in $10 \%$ SDS-PAGE gel. Phosphorylated MAPKs were detected using an anti-phospho-MAP kinase (p42/44) antibody (Cell Signaling Technology, USA) as previously described (Sharma and Carew, 2002).

\section{RESULTS AND DISCUSSION}

The $m k k 3$ mutant has a developmental phenotype depending on the day length

Plant genomes contain a huge number of MAPK cascade genes compared to those of other eukaryotes including, yeast and human. Although the plant MKK gene family has relatively 
A

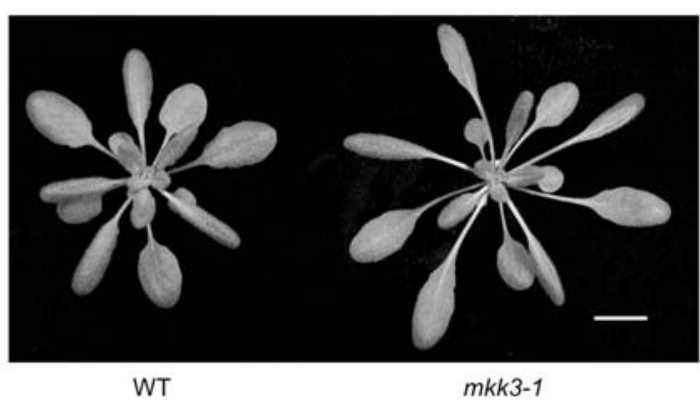

B
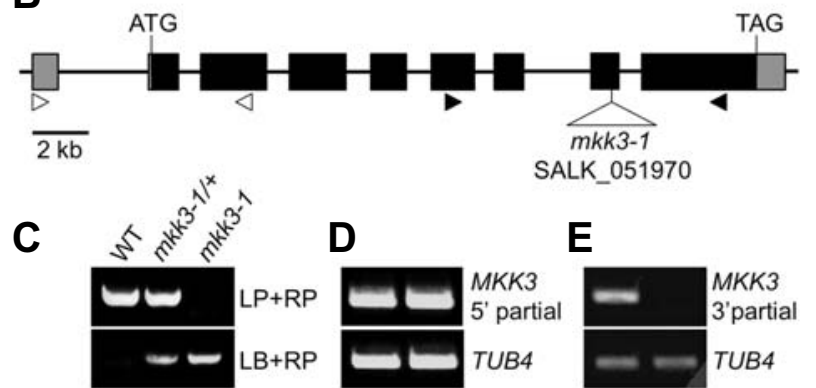

E

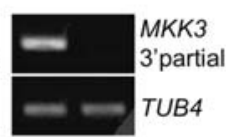

Fig. 1. The phenotype of the $m k k 3-1$ mutant is co-segregated with the T-DNA insertion. (A) Arabidopsis WT and $m k k 3-1$ plants were grown in soil at $22^{\circ} \mathrm{C}, 65 \%$ humidity, and $80 \mu \mathrm{mol} \mathrm{m}^{-2} \mathrm{~s}^{-1}$ light intensity under a $12 \mathrm{~h}$ photoperiod in a growth room. Scale bar, $10 \mathrm{~mm}$. (B) The genome structure of MKK3. Gray color boxes, 5'- or 3'untranslated regions. Black color boxes, exons. Lines between boxes, introns. T-DNA is inserted at the $7^{\text {th }}$ exon of the $M K K 3$ gene in SALK_051970. (C) Genotype analysis of WT, heterozygous, and homozygous plants. The SALK_051970 line was identified using LP, $\mathrm{RP}$, and LB primers obtained from the T-DNA primer design program of SIGnAL. (D, E) Semi-quantitative RT-PCR of MKK3 was performed using different sets of primers. White arrowheads, forward and reverse primers for $5^{\prime}$ partial MKK3 (D). Black arrowheads, forward and reverse primers for $3^{\prime}$ partial $M K K 3(E)$.

few members from MKK1 to MKK10 among MAPK cascade gene families, they genetically display the functional redundancies in various signaling pathways (Gao et al., 2008; Lampard et al., 2014; Pitzschke et al., 2009; Teige et al., 2004). Hence, it is very hard for a single $m k k$ mutant to have a specific phenotype under normal growth conditions. However, I found one mkk3 mutant with a striking phenotype showing a long petiole in leaf development (Fig. 1A), which was initially obtained from the ABRC (Fig. 1B). Interestingly, this phenotype was stronger in shorter day lengths and developmental stages (Supplementary Fig. 1) than in long day conditions (data not shown). In addition, I also observed the additional phenotype of yellowish leaf veins (chlorosis around leaf veins) (Supplementary Fig. 2). To eliminate the possibility of multiple mutations, backcrossing the mkk3 mutant (Salk_051970) with the WT was performed. As expected, none of the $F_{1}$ plants showed any phenotype (Fig. $1 \mathrm{C}$ and Supplementary Fig. 3), and when self-pollinated, they then produced $F_{2}$ plants showing the segregation of phenotypes. For instance, under the homozygous recessive $m k k 3$ mutant background, plants were still segregated into approximately 3:1 ratio for the yellowish leaf vein phenotype (WT leaf vein : yellowish leaf vein $=58: 22, \chi^{2}=0.217, p<0.5$ ), which was not correlated with the T-DNA insertion at the $7^{\text {th }}$ exon of
A
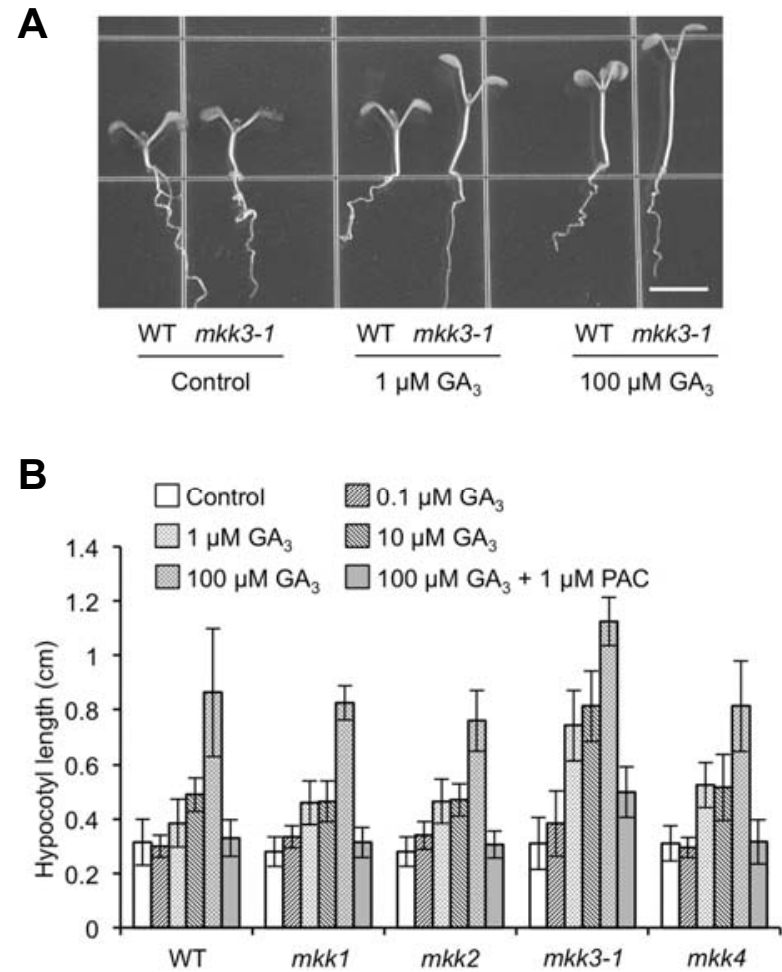

Fig. 2. The $m k k$ 3-1 mutant shows GA-hypersensitive responses. (A) Arabidopsis WT and $m k k 3-1$ plants were vertically grown on MS plates with 1 and $100 \mu \mathrm{M} \mathrm{GA}_{3}$ for 7 days under long-day conditions. Scale bar, $5 \mathrm{~mm}$. (B) Arabidopsis WT, $m k k 1, m k k 2, m k k 3-1$, and $m k k 4$ plants were vertically grown on MS plates with 1,10 , or 100 $\mu \mathrm{M} \mathrm{GA}{ }_{3}$ or $1 \mu \mathrm{M}$ paclobutrazol (PAC), a GA biosynthetic inhibitor. Error bars, s.d. $(n=10)$.

the MKK3 gene (Figs. 1B and 1C; Supplementary Fig. 4). Although $5^{\prime}$ partial transcripts of $M K K 3$ were still abundant (Fig. 1D), the full-length transcript of $M K K 3$ was completely wiped out by T-DNA insertion (Figs. $1 \mathrm{~B}$ and $1 \mathrm{E}$ ). In the end, the TDNA insertion of the Salk_051970 line was revealed to cosegregate only with the long petiole phenotype in intermediate light conditions (12 $\mathrm{h}$ light/12 $\mathrm{h}$ dark photoperiod), which hereafter refers to $m k k 3-1$ based on a previous report (Takahashi et al., 2007). However, the mkk3-1 phenotype should be reproduced by another allele before finally claiming that $m k k 3-1$ caused the enhanced growth in hypocotyls and petioles.

The mkk3-1 mutant displays a GA-hypersensitive phenotype in hypocotyl elongation

Phytohormone GA regulates hypocotyl elongation and the repression of light-regulated genes, which usually resembles the etiolated responses of seedlings grown in darkness (Alabadí et al., 2004). In addition to the long petiole phenotype shown in mkk3-1 mutant plants, they also displayed a GA-hypersensitive phenotype on hypocotyl elongation (Fig. 2A). Among the $m k k$ mutants, surprisingly, only mkk3-1 seedlings showed longer hypocotyl lengths in the treatment of exogenous $\mathrm{GA}_{3}$ (Fig. $2 \mathrm{~B}$ and Supplementary Fig. 5) and hypocotyl elongation was proportionally increased depending on the concentrations of exogenous $\mathrm{GA}_{3}$ (Fig. 2). Since the elongated hypocotyl length of the mkk3-1 mutant was retained in the presence of paclobutrazol 
A

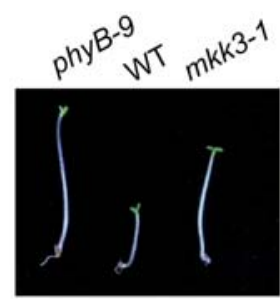

Red Light

B

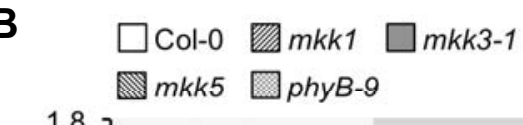

Fig. 3. The $m k k 3-1$ mutant is partially impaired in red light signaling. (A) The $m k k 3-1$ mutant showed a less sensitive response to red light signals in the hypocotyl elongation. Arabidopsis WT, phyB-9, and $m k k 3-1$ plants were vertically grown on MS plates for 4 days at $22^{\circ} \mathrm{C}$ under red light conditions $\left(7 \mu \mathrm{mol} \mathrm{m} \mathrm{m}^{-2} \mathrm{~s}^{-1}\right.$ light intensity) in the growth chamber. (B) The mkk3-1 mutant showed longer hypocotyl length in red light conditions but other $m k k 1$ and $m k k 5$ mutants did not. Hypocotyl length was measured using the Image $\mathrm{J}$ program. RL-S, red light without sucrose. D-S, dark without sucrose. $R L+S$, red light with $1 \%$ sucrose. D+S, dark with $1 \%$ sucrose. Error bars, s.d. $(n=10)$.

(PAC), an inhibitor of GA biosynthesis (Fig. 2B), this suggests that other regulatory mechanisms are also required for the coordinated regulation of the hypocotyl elongation shown in the $m k k 3-$ 1 mutant. To investigate the possibility of MAPK activation in GA signaling, MAPK phosphorylation was examined. However, I did not observe any significant activation of MAPKs, when the Arabidopsis protoplast system was applied with the treatment of exogenous $100 \mu \mathrm{M} \mathrm{GA}_{3}$ (Supplementary Fig. 6). Thus, it suggests that the role of MAPK cascades in GA signaling is probably mediated through the sharing of common downstream target(s).

The mkk3-1 mutant shows a less sensitive phenotype in light signaling

Because the phenotypes of elongated hypocotyls and petioles usually resemble those of the phyB mutant (Figs. $1 \mathrm{~A}$ and $2 \mathrm{~A}$ ) (Reed et al., 1993), I tested whether the lesion of the $m k k 3-1$ mutant is correlated with red light signaling. As shown in Fig. $3 A$, the WT seedling showed photomorphogenic growth in red light conditions, whereas this response of the mkk3-1 mutant was strongly impaired like the phyB-9 mutant, demonstrating that $M K K 3$ is probably involved in red light-mediated signaling pathway. Moreover, this less sensitive phenotype was only shown in the mkk3-1 mutant among other mkk mutants (Fig. 3B). In contrast, it has recently been reported that hypocotyl
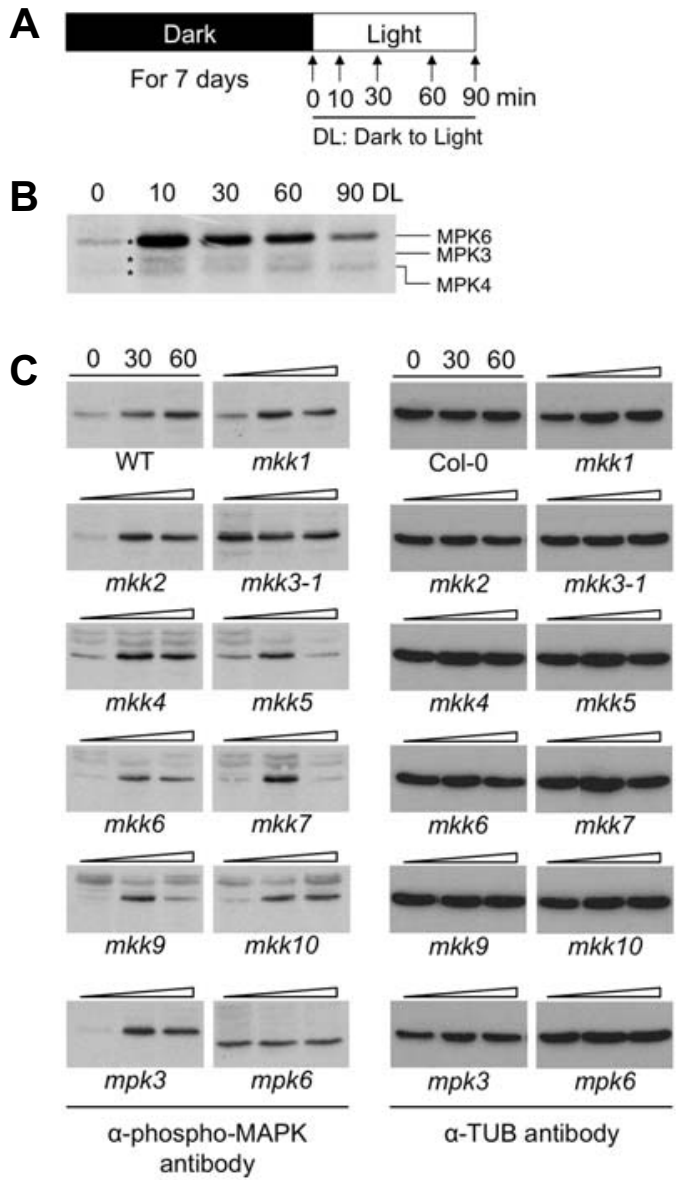

Fig. 4. Light-induced MAPK activation is affected in the $m k k 3-1$ mutant. (A) A scheme for analyzing light-induced MAPK activation. Seedlings grown in the dark for 7 days were sampled at 10,30,60, or $90 \mathrm{~min}$ after exposure to white light. (B) MAPKs were activated by light signals. Endogenously activated MAPKs were detected in SDS-PAGE gel containing MBP, a general MAPK substrate. Asterisks, phosphorylated MAPKs. (C) Constitutive MAPK activation was shown in $m k k 3-1$ and $m p k 6$ mutant seedlings. MAPK activation was detected by an anti-phospho-MAPK antibody. An anti-TUB antibody was used to compare the quantity of loading amounts. Triangles indicate 0,30 , and $60 \mathrm{~min}$.

lengths of the mkk3-1 mutant did not differ from those of WT grown in red light conditions (Sethi et al., 2014). However, since the hypocotyl elongation in the previous report was examined with relatively higher fluence rate $\left(60 \mu \mathrm{mol} \mathrm{m} \mathrm{m}^{-2} \mathrm{~s}^{-1}\right)$ compared to the condition $\left(7 \mu \mathrm{mol} \mathrm{m} \mathrm{m}^{-2} \mathrm{~s}^{-1}\right)$ used in this study, sensitive hypocotyl responses of the $m k k 3-1$ mutant in red light conditions may be affected by different fluence rates. Indeed, hypocotyl growth is strongly suppressed depending on the increase of fluence rate (Carvalho and Folta, 2014; Oh et al., 2012). Therefore, photomorphogenic responses of the mkk3-1 mutant in different light conditions and intensities must be further elucidated.

MAPKs are activated by light signals

To assess the possible role of MAPK cascades in light signaling, I first tested the MAPK phosphorylation activated by light signals. For this, seedlings were grown in darkness for 7 days 
and exposed to white light for 10, 30,60, and 90 min (Fig. 4A). Interestingly, endogenous MAPKs were phosphorylated from $10 \mathrm{~min}$ and gradually disappeared, which is the general behavior of the MAPK phosphorylation stimulated by input signals (Fig. 4B). Consistent with a previous report (Sethi et al., 2014), MPK6 seemed to be mainly activated and MPK3 and MPK4 were also slightly phosphorylated by light signals (Fig. 4B). In the same context, five other mkk mutants, namely $m k k 1, m k k 2$, $m k k 4, m k k 6$, and $m k k 10$, showed very similar patterns of MAPK phosphorylations detected in WT seedlings, whereas the kinetics of MAPK phosphorylations was accelerated in $m k k 5, m k k 7$, and $m k k 9$ (Fig. 4C). Unlike other mkk mutants, constitutive MPK6 phosphorylations were consistently observed in the mkk3-1 mutant displayed under dark conditions (Fig. 4C) (Sethi et al., 2014), which might be not affected by endogenous levels of MPK6 proteins (Supplementary Fig. 7). Although the mkk3-1 mutant showed similar hypocotyl elongation like the phyB-9 mutant (Fig. 3A), MAPKs in the phyB-9 mutant were still phosphorylated by light signals shown in WT seedlings (Supplementary Fig. 8). This suggests that a less sensitive phenotype of the mkk3-1 mutant in red light signaling seems to be indirectly affected by phyB.

Meanwhile, MPK3 was also constitutively phosphorylated in the mpk6 mutant, whereas a similar pattern of light-induced MPK6 phosphorylation was still observed in the mpk3 mutant (Fig. 4C). The activation of MPK3 and MPK6 is usually enhanced when the other MPK is mutated to compensate for the missing functions (Wang et al., 2008). Because MPK3 and MPK6 are closely related for sharing redundant functions in various signaling pathways, this data shows that MPK3 was presumably involved in light signaling in the absence of MPK6.

Although the MKK3-MPK6 cascade module has recently been reported to be specifically activated by blue light signals (Sethi et al., 2014), as shown in this study, MKK3 also seems to play a crucial role in red light signaling (Fig. 3) and in the negative regulation of MAPK phosphorylation in darkness (Fig. 4C). Moreover, we still cannot rule out the redundant involvement of other MKKs in addition to MKK3. Therefore, this finding sheds new light on the complicated function of MAPK cascades during dark-light transition.

In 2008, Deng's and Prat's groups showed that the crosstalk between light and GA is regulated by the destabilization of PIF proteins that bind to their target promoter and regulate gene expression related with the cell elongation (de Lucas et al., 2008; Feng et al., 2008). Interestingly, since the mkk3-1 mutant has both phenotypes on light and GA responses (Figs. 2 and 3), it is therefore hypothesized that light-mediated MAPK signaling presumably regulates PIF members directly or indirectly. Although the light-mediated regulation of genes such as ELF4 and HY5 was impaired in the mkk3-1 mutant (Supplementary Fig. 9A) (Molas et al., 2006; Sethi et al., 2014), since the transcript levels of PIF genes such as PIF3 and PIF4 were not significantly changed between WT and mkk3-1 plants (Supplementary Fig. 9B), future research should investigate how the stability of $\mathrm{PIF}$ proteins is regulated by ubiquitin-proteasome-mediated degradation through the phosphorylation caused by MAPK cascades. Furthermore, the light-signal-induced subcellular localization of MPK3 and MPK6 should be studied.

Note: Supplementary information is available on the Molecules and Cells website (www.molcells.org).

\section{ACKNOWLEDGMENTS}

I appreciate J Sheen's encouragement for this study and I also thank KH Choi, OK Cha and ME Kim for their technical support.
I thank I Lee for sharing the phyB-9 mutant. This study was supported by a grant (No. 3000002167) from Duksung Women's University.

\section{REFERENCES}

Alabadí, D., Gil, J., Blázquez, M.A., and García-Martínez, J.L. (2004). Gibberellins repress photomorphogenesis in darkness. Plant Physiol. 134, 1050-1057.

Asai, T., Tena, G., Plotnikova, J., Willmann, M.R., Chiu, W.L., Gomez-Gomez, L., Boller, T., Ausubel, F.M., and Sheen, J. (2002). MAP kinase signalling cascade in Arabidopsis innate immunity. Nature 415, 977-983.

Bu, Q., Zhu, L., Dennis, M.D., Yu, L., Lu, S.X., Person, M.D., Tobin E.M., Browning, K.S., and Huq, E. (2011). Phosphorylation by CK2 enhances the rapid light-induced degradation of phytochrome interacting factor 1 in Arabidopsis. J. Biol. Chem. 286, 12066-12074.

Carvalho, S.D., and Folta, K.M. (2014). Sequential light programs shape kale (Brassica napus) sprout appearance and alter metabolic and nutrient content. Hort. Res. 1, 8.

Chen, M., and Chory, J. (2011). Phytochrome signaling mechanisms and the control of plant development. Trends Cell Biol. 21, 664-671.

Dai, Y., Wang, H., Li, B., Huang, J., Liu, J., Liu, X., Zhou, Y., Mou, Z., and Li, J. (2006). Increased expression of MAPK kinase kinase7 causes deficiency in polar auxin transport and leads to plant architectural abnormality in Arabidopsis. Plant Cell 18, 308-320.

de Lucas, M., Jean-Michel, D., Rodríguez-Falcón, M., Pontin, M., Iglesias-Pedraz, J.M., Lorrain, S., Fankhauser, C., Blázquez, M.A., Titarenko, E., and Prat, S. (2008). A molecular framework for light and gibberellin control of cell elongation. Nature 451, 480-484

Dóczi, R., Brader, G., Pettkó-Szandtner, A., Rajh, I., Djamei, A., Pitzschke, A., Teige, M., and Hirt, H. (2007). The Arabidopsis mitogen-activated protein kinase kinase MKK3 is upstream of group $\mathrm{C}$ mitogen-activated protein kinases and participates in pathogen signaling. Plant Cell 19, 3266-3279.

Feng, S., Martinez, C., Gusmaroli, G., Wang, Y., Zhou, J., Wang, F., Chen, L., Yu, L., Iglesias-Pedraz, J.M., Kircher, S., et al. (2008). Coordinated regulation of Arabidopsis thaliana development by light and gibberellins. Nature 451, 475-479.

Gao, M., Liu, J., Bi, D., Zhang, Z., Cheng, F., Chen, S., and Zhang, Y. (2008). MEKK1, MKK1/MKK2 and MPK4 function together in a mitogen-activated protein kinase cascade to regulate innate immunity in plants. Cell Res. 18, 1190-1198.

Hamel, L.P., Nicole, M.C., Sritubtim, S., Morency, M.J., Ellis, M., Ehlting, J., Beaudoin, N., Barbazuk, B., Klessig, D., Lee, J., et al. (2006). Ancient signals: comparative genomics of plant MAPK and MAPKK gene families. Trends Plant Sci. 11, 192-198.

Ichimura, K., Shinozaki, K., Tena, G., Sheen, J., Henry, Y., Champion, A., Kreis, M., Zhang, S., Hirt, H., Wilson, C., et al. (2002). Mitogen-activated protein kinase cascades in plants: a new nomenclature. Trends Plant Sci. 7, 301-308.

Jang, I.C., Yang, J.Y., Seo, H.S., and Chua, N.H. (2005). HFR1 is targeted by COP1 E3 ligase for post-translational proteolysis during phytochrome A signaling. Genes Dev. 19, 593-602.

Lampard, G.R., Lukowitz, W., Ellis, B.E., and Bergmann, D.C. (2009). Novel and expanded roles for MAPK signaling in Arabidopsis stomatal cell fate revealed by cell type-specific manipulations. Plant Cell 21, 3506-3517.

Lampard, G.R., Wengier, D.L., and Bergmann, D.C. (2014). Manipulation of mitogen-activated protein kinase kinase signaling in the Arabidopsis stomatal lineage reveals motifs that contribute to protein localization and signaling specificity. Plant Cell 26, 33583371.

Leivar, P., and Quail, P.H. (2010). PIFs: pivotal components in a cellular signaling hub. Trends Plant Sci. 16, 19-28.

Matsuoka, D., Nanmori, T., Sato, K.I., Fukami, Y., Kikkawa, U., and Yasuda, T. (2002). Activation of AtMEK1, an Arabidopsis mitogen-activated protein kinase kinase, in vitro and in vivo: analysis of active mutants expressed in E. coli and generation of the active form in stress response in seedlings. Plant J. 29, 637-647.

Melikant, B., Giuliani, C., Halbmayer-Watzina, S., Limmongkon, A., 
Heberle-Bors, E., and Wilson, C. (2004). The Arabidopsis thaliana MEK AtMKK6 activates the MAP kinase AtMPK13. FEBS Lett. 576, 5-8.

Molas, M.L., Kiss, J.Z., and Correll, M.J. (2006). Gene profiling of the red light signaling pathways in roots. J. Exp. Bot. 57, 32173229.

Oh, E., Zhu, J.Y., and Wang, Z.Y. (2012). Interaction between BZR1 and PIF4 integrates brassinosteroid and environmental responses. Nat. Cell Biol. 14, 802-809.

Park, H.J., Ding, L., Dai, M., Lin, R., and Wang, H. (2008). Multisite phosphorylation of Arabidopsis HFR1 by casein kinase II and plausible role in regulating its degradation rate. J. Biol. Chem. 283, 23264-23273.

Pitzschke, A., Djamei, A., Bitton, F., and Hirt, H. (2009). A major role of the MEKK1-MKK1/MKK2-MPK4 pathway in ROS signalling. Mol. Plant 2, 120-137.

Reed, J.W., Nagpal, P., Poole, D.S., Furuya, M., and Chory, J. (1993). Mutations in the gene for the red/far-red light receptor phytochrome $\mathrm{B}$ alter cell elongation and physiological responses throughout Arabidopsis development. Plant Cell 5, 147-157.

Sajio, Y., Sullivan, J.A., Wang, H. Yang, J., Shen, Y., Rubio, V., Ma, L., Hoecker, U., and Deng, X.W. (2003). The COP1-SPA1 interaction defines a critical step in phytochrome A-mediated regulation of HY5 activity. Genes Dev. 17, 2642-2647.

Seo, H.S., Yang, J.Y., Ishikawa, M., Bolle, C., Ballestros, M.L., and Chua, N.H. (2003). LAF1 ubiquitination by COP1 controls photomorphogenesis and is stimulated by SPA1. Nature 423 , 995-999.

Sethi, V., Raghuram, B., Sinha, A.K., and Chattopadhyay, S. (2014). A mitogen-activated protein kinase cascade module, MKK3MPK6 and MYC2, is involved in blue light-mediated seedling development in Arabidopsis. Plant Cell 26, 3343-3357.

Sharma, S.K., and Carew, T.J. (2002). Inclusion of phosphatase inhibitors during western blotting enhances signal detection with phospho-specific antibodies. Anal. Biochem. 307, 187-189.

Soyano, T., Nishihama, R., Morikiyo, K., Ishikawa, M., and Machida, Y. (2003). NQK1/NtMEK1 is a MAPKK that acts in the NPK1 MAPKKK-mediated MAPK cascade and is required for plant cytokinesis. Genes Dev. 17, 1055-1067.

Takahashi, F., Yoshida, R., Ichimura, K., Mizoguchi, T., Seo, S. Yonezawa, M., Maruyama, K., Yamaguchi-Shinozaki, K., and Shinozaki, K. (2007). The mitogen-activated protein kinase cascade MKK3-MPK6 is an important part of the jasmonate signal transduction pathway in Arabidopsis. Plant Cell 19, 805-818.

Teige, M., Scheikl, E., Eulgem, T., Dóczi, R., Ichimura, K., Shinozaki, K., Dangl, J.L., and Hirt, H. (2004). The MKK2 pathway mediates cold and salt stress signaling in Arabidopsis. Mol. Cell 15, 141-152.

Wang, H., Ngwenyama, N., Liu, Y., Walker, J.C., and Zhang, S. (2007). Stomatal development and patterning are regulated by environmentally responsive mitogen-activated protein kinase in Arabidopsis. Plant Cell 19, 63-73.

Wang, H., Liu, Y., Bruffett, K., Lee, J., Hause, G., Walker, J.C., and Zhang, S. (2008). Haplo-insufficiency of MPK3 in MPK6 mutant background uncovers a novel function of these two MAPKs in Arabidopsis ovule development. Plant Cell 20, 602-613.

Yang, J., Lin, R., Sullivan, J., Hoecker, U., Liu, B., Xu, L., Deng, X.W., and Wang, H. (2005). Light regulates COP1-mediated degradation of HFR1, a transcription factor essential for light signaling in Arabidopsis. Plant Cell 17, 804-821.

Yoo, S.D., Cho, Y.H., Tena, G., Xiong, Y., and Sheen, J. (2008). Dual control of nuclear EIN3 by bifurcate MAPK cascades in $\mathrm{C}_{2} \mathrm{H}_{2}$ signalling. Nature 451, 789-795.

Zhang, S., and Klessig, D.F. (1997). Salicylic acid activates a 48-kD MAP kinase in tobacco. Plant Cell 9, 809-824. 